University of Nebraska - Lincoln

DigitalCommons@University of Nebraska - Lincoln

$9-2003$

\title{
Two semi-infinite interfacial cracks between two bonded dissimilar elastic strips
}

\author{
Xiangfa Wu \\ Department of Engineering Mechanics, University of Nebraska-Lincoln, xfwu@unlserve.unl.edu
}

Yuris A. Dzenis

Department of Engineering Mechanics,University of Nebraska-Lincoln, ydzenis@unl.edu

Tian-You Fan

Beijing Institute of Technology

Follow this and additional works at: https://digitalcommons.unl.edu/engineeringmechanicsfacpub

Part of the Mechanical Engineering Commons

Wu, Xiangfa; Dzenis, Yuris A.; and Fan, Tian-You, "Two semi-infinite interfacial cracks between two bonded dissimilar elastic strips" (2003). Faculty Publications from the Department of Engineering Mechanics. 31. https://digitalcommons.unl.edu/engineeringmechanicsfacpub/31

This Article is brought to you for free and open access by the Mechanical \& Materials Engineering, Department of at DigitalCommons@University of Nebraska - Lincoln. It has been accepted for inclusion in Faculty Publications from the Department of Engineering Mechanics by an authorized administrator of DigitalCommons@University of Nebraska - Lincoln. 
Published in International Journal of Engineering Science 41:15 (September 2003), pp. 1699-1710;

doi 10.1016/S0020-7225(03)00107-1 Copyright @ 2003 Elsevier Science Ltd. Used by permission.

http://www.elsevier.com/wps/product/cws_home/278

Submitted October 16, 2002; accepted February 17, 2003; published online April 18, 2003.

\title{
Two semi-infinite interfacial cracks between two bonded dissimilar elastic strips
}

\author{
Xiang-Fa Wu, ${ }^{a}$ Yuris A. Dzenis, ${ }^{a}$ and Tian-You Fan ${ }^{b}$ \\ a Department of Engineering Mechanics, Center for Materials Research and Analysis, \\ University of Nebraska-Lincoln, Lincoln, NE 68588-0526, USA \\ ${ }^{b}$ Department of Applied Physics, College of Science, Beijing Institute of Technology, Beijing 100081, China \\ Corresponding author - X.-F. Wu, email xfwu@unlserve.unl.edu; fax 402 472-8292
}

\begin{abstract}
The complex stress intensity factor and energy release rate are obtained for two semi-infinite interfacial cracks between two bonded dissimilar elastic strips with equal thickness under inplane deformations. During the procedure, by means of conformal mapping technique, the mixed boundary-value problem is reduced to a standard Riemann-Hilbert problem, which is further solved in closed-form. In some limiting cases, the present explicit solutions can cover the well-known results in the literature.
\end{abstract}

Keywords: interfacial crack, bimaterial strip, stress intensity factor, Riemann-Hilbert problem, conformal mapping, closed-form solution

\section{Introduction}

Since the pioneering work by Williams [1], a substantial progress has been made on the analysis of interfacial fracture in elastic bimaterials. Investigators have contributed significantly in this interesting subject in the last four decades, such as the early work by England [2], Erdogan [3], Rice and Sih [4], Cherepanov [5], and Rice [6], etc. A thorough study has been conducted on the characteristics of the asymptotic fields near crack tip and the stress intensity factor (SIF) and energy release rate (ERR) of interfacial cracks between two bonded dissimilar half-planes. Systematic fracture criteria and interfacial fracture test methods have been established. In practice, interfacial cracks between two bonded dissimilar strips are very common in engineering structures such as composite 
laminates and adhesive joints etc., where cracks often bear the length comparable to the structure dimensions. In order to evaluate the interaction of structure boundaries and cracks, it is natural to introduce the structure dimensions into the crack model. A typical crack model developed by investigators is concerned with a semi-infinite interfacial crack between two bonded dissimilar strips with equal thickness. Its antiplane case is mathematically equivalent to that of a semi-infinite crack in a homogenous elastic strip, which was discussed by Rice [7] using J-integral, Georgiadis [8] using the Wiener-Hopf technique, and Li [9] using dual integral equations. Recently, Wu and Dzenis [10] also considered an antiplane interfacial edge crack between two bonded dissimilar semi-infinite elastic strips using the known screw dislocation solution combined with conformal mapping technique. The obtained SIF solution could be used to correct the beam theory utilized in evaluation of the SIF and ERR of bimaterials in the double cantilever beam test configuration. In the case of a semi-infinite inplane crack in an elastic strip, Fan [11-13] obtained the SIF and the size of plastic zone solution by means of Muskhelishvili's method [14]. Furthermore, Fan [15] and Maruyama and Fan [16] extended their strip crack model into seismology to analyze the breakdown zone in a fault. Recently, Shen and Fan [17] further considered two semi-infinite cracks in an elastic strip using the above method. In the case of inplane interfacial cracks in bimaterial strips, a number of important results have been achieved mainly based on the elementary beam theory. Recent refined work on the basis of J-integral and the asymptotic fields near crack tip has been conducted by Suo and Hutchinson [18-19], Suo [20], and Bao et al. [21]. A series of semi-analytic solutions involving various crack configurations were provided in their work. Wu et al. [22] also provided an explicit solution for a semi-infinite interfacial crack in a bimaterial strip by reducing the problem to a standard Riemann-Hilbert problem.

In this paper, we consider the problem of two semi-infinite interfacial cracks in a bimaterial strip under inplane deformations, which is the extension of the work by Shen and Fan [17] and Wu et al. [22]. By means of conformal mapping technique, the present mixed boundary-value problem is reduced to a standard Riemann-Hilbert problem, which is then solved explicitly. The complex SIF and ERR are given in closed-form. Furthermore, the SIFs of some special cases with one (two) semi-infinite crack(s) between two bonded dissimilar half-planes or in a homogeneous strip can be extracted as the limiting cases of the strip dimensions and elastic properties, respectively.

\section{Problem statement and solution}

As shown in Figure 1(a), we consider two dissimilar elastic strips with equal thickness $H$ are bonded along straight-line segments along $0<x<L$ at the $x$-axis. Assume a material with elastic properties $E_{1}$ and $v_{1}$ occupy the upper strip, $0<y<H$, and a material with elastic properties $E_{2}$ and $v_{2}$ occupy the lower strip, $-H<y<0$. In the following, for convenience of the derivation, all quantities such as the elastic constants, stresses, and so on, relating to the region $0<y<H$ and $-H<y<0$ will be marked with subscripts 1 and 2, respectively.

Without loss of generality, we assume the crack surfaces with the action of self-equilibrated forces, normal force $p(x)$ and shear $q(x)$, and the upper and lower strip surfaces stress-free. The boundary conditions and the stress/displacement continuity along the interface of the bonded strips may be expressed as 


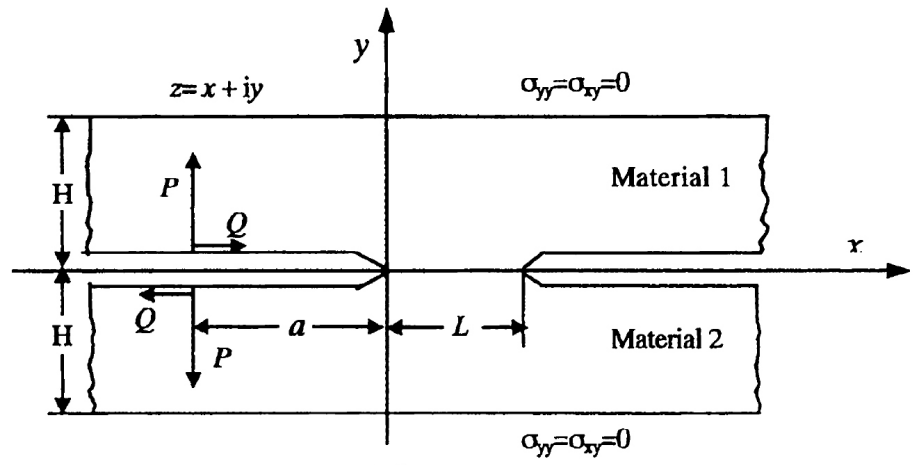

(a)

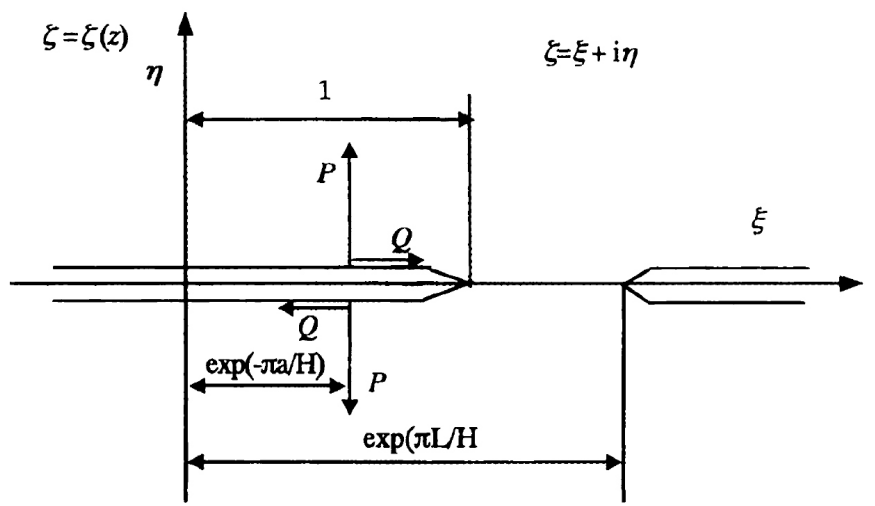

(b)

Figure 1. Two semi-infinite interfacial cracks in a bimaterial system: (a) two bonded dissimilar strips and (b) two bonded dissimilar half-planes.

$$
\begin{aligned}
& y= \pm H,-\infty<x<+\infty: \sigma_{y y}=\sigma_{x y}=0, \\
& x= \pm \infty,-H<y<H: \sigma_{x x}=\sigma_{x y}=0, \\
& y=0,-\infty<x<0 \text { or } L<x<+\infty: \sigma_{y y}=p(x), \sigma_{x y}=q(x), \\
& y=0,0<x<L: \sigma_{y y}\left(x, 0^{+}\right)=\sigma_{y y}\left(x, 0^{-}\right), \sigma_{x y}\left(x, 0^{+}\right)=\sigma_{x y}\left(x, 0^{-}\right), \\
& y=0,0<x<L: u_{y}\left(x, 0^{+}\right)=u_{y}\left(x, 0^{-}\right), u_{x}\left(x, 0^{+}\right)=u_{x}\left(x, 0^{-}\right) .
\end{aligned}
$$

In interfacial fracture mechanics, two Dundurs' parameters [23] are often introduced to simplify the derivation, which are defined as follows:

$$
\alpha=\frac{\Gamma\left(\kappa_{2}+1\right)-\left(\kappa_{1}+1\right)}{\Gamma\left(\kappa_{2}+1\right)+\left(\kappa_{1}+1\right)}, \quad \beta=\frac{\Gamma\left(\kappa_{2}-1\right)-\left(\kappa_{1}-1\right)}{\Gamma\left(\kappa_{2}+1\right)+\left(\kappa_{1}+1\right)},
$$

where $\Gamma=\mu_{1} / \mu_{2}, \kappa_{j}=3-4 v_{j}$ for plane strain and $\left(3-v_{j}\right) /\left(1+v_{j}\right)$ for plane stress, $\mu_{j}(j=1,2)$ is the material shear modulus, and $v_{j}(j=1,2)$ is the Poisson's ratio. The physically admissible values of $\alpha$ and $\beta$ are restricted to a parallelogram enclosed by $\alpha= \pm 1$ and $\alpha-4 \beta= \pm 1$ in the $\alpha, \beta$-plane, and the two parameters measure the elastic dissimilarity of two materials in the sense that both vanish when the dis- 
similarity does. There are two other bimaterial parameters, $\Sigma$, the stiffness, and $\varepsilon$, the oscillatory index, relating to $\alpha$ and $\beta$, respectively, by

$$
\Sigma=\frac{c_{2}}{c_{1}}=\frac{1+\alpha}{1-\alpha}, \quad \varepsilon=\frac{1}{2 \pi} \ln \frac{1-\beta}{1+\beta},
$$

where $c_{j}=\left(\kappa_{j}+1\right) / \mu_{j^{\prime}}(j=1,2)$, is a measure of the compliance of a material. Thus $\alpha$ can be further interpreted as a measure of the dissimilarity in stiffness of the two materials with material 1 stiffer than 2 as $\alpha>0$, while material 1 relatively compliant as $\alpha<0$. Generally, $\varepsilon$ is very small, and in this paper our purpose is to find the SIF and ERR solutions mathematically and no special attention is paid to such effects as crack faces contact due to the nonzero $\varepsilon$, which were discussed by Comnimou [25-27], Rice [6], and others.

Due to the mismatch of material properties of the two component materials, the stress field near the interfacial crack tip bears a $r^{-1 / 2+i \varepsilon}$ type singularity. The nonzero index $\varepsilon$ leads to the oscillatory feature of elastic field near the crack tip. This phenomenon causes confusion about the definitions of the corresponding fracture parameters. By introducing an intrinsic material length scale $l_{0}$ Rice [6] defined a complex SIF with the same unit as the classic SIF of cracks in homogenous materials, $K=K_{1}+i K_{2}$, in which the magnitude of $\mathbf{K}$ does not vary with different choices of $l_{0}$ while its phase angle may change. With the complex SIF defined by Rice [6], the traction on the interface a distance $r$ ahead of the crack tip is expressed as

$$
\sigma_{y y}+\mathrm{i} \sigma_{x y}=\frac{\mathbf{K}}{\sqrt{2 \pi r}}\left(\frac{r}{l_{0}}\right)^{\mathrm{i} \varepsilon}
$$

the relative crack opening displacements a distance $r$ behind the crack tip are

$$
\delta_{y}+\mathrm{i} \delta_{x}=\frac{c_{1}+c_{2}}{2 \sqrt{2 \pi}(1+\mathrm{i} \varepsilon) \cosh (\pi \varepsilon)} \mathbf{K} \sqrt{r}\left(\frac{r}{l_{0}}\right)^{\mathrm{i} \varepsilon},
$$

and the ERR is evaluated as

$$
G=\frac{c_{1}+c_{2}}{16 \cosh ^{2}(\pi \varepsilon)}|\mathbf{K}|^{2}
$$

Hereafter we will derive the closed-form solution by reducing the aforementioned mixed boundary-value problem to a standard Riemann-Hilbert problem, which has been discussed extensively by Muskhelishvili [14] and further used for finding the SIF solutions to collinear interfacial cracks by England [2], Erdogan [3], Rice and Sih [4], and recently by Suo [24]. Consider the complex expressions of the stress and displacement components in terms of the complex potentials. The stresses and displacements for each homogenous medium under inplane deformations may be represented by two Muskhelishvili's complex potentials $\phi_{j}(z)$ and $\psi_{j}(z), j=1,2$, of the variable $z=x+\mathrm{i} y$ [14], which can be further simplified by introducing a pair of commonly used potentials, $\Phi(z)$ and $\Psi(z)$, defined as 


$$
\Phi(z)=\phi^{\prime}(z), \quad \Omega(z)=\left[z \phi^{\prime}(z)+\psi(z)\right]^{\prime} .
$$

Therefore, the stress and displacement components can be derived from the following relations:

$$
\begin{aligned}
& \sigma_{x x}+\sigma_{y y}=2[\Phi(z)+\overline{\Phi(z)}], \\
& \sigma_{y y}+\mathrm{i} \sigma_{x y}=\overline{\Phi(z)}+\Omega(z)+(\bar{z}-z) \Phi^{\prime}(z), \\
& -2 \mathrm{i} \mu \frac{\partial}{\partial x}\left(u_{y}+\mathrm{i} u_{x}\right)=\kappa \overline{\Phi(z)}-\Omega(z)-(\bar{z}-z) \Phi^{\prime}(z) .
\end{aligned}
$$

We first introduce the governing equations of colinear interfacial cracks between two bonded dissimilar elastic half-planes with the action of self-equilibrated forces at crack surfaces as discussed by Suo [24]. Let the potentials in this case be written as

$$
\Phi(z)=\left\{\begin{array}{ll}
\Phi^{a}(z), & z \in D_{1} \\
\Phi^{b}(z), & z \in D_{2}
\end{array}, \quad \Omega(z)= \begin{cases}\Omega^{a}(z), & z \in D_{1} \\
\Omega^{b}(z), & z \in D_{2}\end{cases}\right.
$$

where the superscripts " $a$ " and " $b$ " indicate the potentials for the half-planes above and below respectively, and $D_{1}$ and $D_{2}$ denote the domains for the half-planes above and below, respectively. The stress continuity of $\sigma_{y y}+\mathrm{i} \sigma_{x y}$ across the interface requires [24]

$$
\begin{aligned}
& \overline{\Phi^{a}(z)}=\Omega^{b}(z), \quad z \in D_{2}, \\
& \overline{\Phi^{b}(z)}=\Omega^{a}(z), \quad z \in D_{1} .
\end{aligned}
$$

Substitution of (10) into (8) leads to the derivative of displacement jumps across the interface or the components of the Burgers vector as

$$
-2 \mathrm{i} \frac{\partial}{\partial x}\left(\delta_{y}+\mathrm{i} \delta_{x}\right)=\frac{c_{1}+c_{2}}{2}\left[(1-\beta) \Omega^{b}(x)-(1+\beta) \Omega^{a}(x)\right] .
$$

Furthermore, due to the displacement continuity across the bonded portions of the interface, an auxiliary function $g(z)$ can be defined to be analytic in the boned whole plane except on the crack lines, such that

$$
\begin{aligned}
& \Omega^{a}(z)=(1-\beta) g(z), z \in D_{1^{\prime}} \\
& \Omega^{b}(z)=(1+\beta) g(z), z \in D_{2} .
\end{aligned}
$$

By inserting (12) into (11), the Burgers vector can be expressed in terms of $g(z)$ as

$$
-2 \mathrm{i} \frac{\partial}{\partial x}\left(\delta_{y}+\mathrm{i} \delta_{x}\right)=\frac{c_{1}+c_{2}}{2}\left(1-\beta^{2}\right)\left[g^{-}(x)-g^{+}(x)\right],
$$

and the traction on the interface can be expressed as

$$
\sigma_{y y}+\mathrm{i} \sigma_{x y}=(1+\beta) g^{-}(x)+(1-\beta) g^{+}(x) .
$$


The prescribed traction on the crack surfaces leads to the following Riemann-Hilbert problem [24]

$$
(1+\beta) g^{-}(x)+(1-\beta) g^{+}(x)=\sigma_{y y}+\mathrm{i} \sigma_{x y} \quad\left(\text { on crack lines } \Gamma=\sum_{i=1}^{n} \Gamma_{i}\right) .
$$

In order to solve the current problem of a bimaterial strip with two stress-free surfaces and two semiinfinite interfacial cracks along the strip mid-plane at the negative $x$-axis and $x \in(L,+\infty)$ at the positive $x$-axis, we introduce the following conformal mapping,

$$
\varsigma=\exp (\pi z / H)
$$

which maps the cracked bimaterial strip, as shown in Figure 1(a), onto two bonded half-planes $(\zeta=$ $\xi+i \eta)$ with two semi-infinite cuts along $\xi \in(-\infty, 1]$ and $\xi \in(\exp (\pi L / H),+\infty]$, as shown in Figure $1(b)$. Here two crack tips $x=0$ and $x=L$ in the bonded strips are mapped onto $\xi=1$ and $\xi=\exp (\pi L / H)$ in the image plane, respectively. Then, using the similar process as discussed by Suo [24], we obtain a new Riemann-Hilbert problem in the whole image plane $(\zeta=\xi+i \eta)$ with two semi-infinite cuts as

$$
(1+\beta) G^{-}(\xi)+(1-\beta) G^{+}(\xi)=\sigma_{\eta \eta}+\mathrm{i} \sigma_{\xi \eta^{\prime}}\{\xi \in(-\infty, 1] \quad \text { and } \quad \xi \in[\exp (\pi L / H),+\infty)\}
$$

Equation (17) includes all the boundary conditions along the crack lines and it also extends to the stress-free conditions of the strip surfaces. Based on the standard method given by Mushhelishvili [14], the homogenous solution of (17) in the $\zeta$-plane may be expressed as

$$
\chi(\varsigma)=(\varsigma-1)^{-1 / 2+i \varepsilon}\left[S^{-} \exp (\pi L / H)\right]^{-1 / 2-i \varepsilon} .
$$

Hence, in the $(\xi, \eta)$-coordinate system the general solution of $(17)$ is written as

$$
G(\varsigma)=\frac{1}{1-\beta} \frac{\chi(\varsigma)}{2 \pi \mathrm{i}} \int_{\Gamma^{\prime}} \frac{\sigma_{\eta \eta}(\xi)+\mathrm{i} \sigma_{\xi \eta}(\xi)}{\chi^{+}(\xi)(\xi-\varsigma)} \mathrm{d} \xi+\chi(\varsigma) P(\varsigma) .
$$

Here the integral should be taken over the union of the two mapped cuts, and $P(S)$ is a polynomial with the form of

$$
P(\mathrm{~s})=\mathrm{C}_{0}+\mathrm{C}_{1} \mathrm{~s}
$$

in which $C_{0}$ and $C_{1}$ are two unknowns to be determined.

Noting that the mapping function (16) maps $x= \pm \infty$ in the physical plane onto $\xi=+\infty$ and 0 in the image plane, respectively, and stresses are bounded at $x= \pm \infty$, we can determine the unknowns $C_{0}$ and $C_{1}$ as 


$$
C_{0}=-\frac{1}{1-\beta} \frac{1}{2 \pi \mathrm{i}} \int_{\Gamma^{\prime}} \frac{\sigma_{\eta \eta}(\xi)+\mathrm{i} \sigma_{\xi \eta}(\xi)}{\chi^{+}(\xi) \xi} \mathrm{d} \xi, \quad C_{1}=0 .
$$

Therefore, substitution of (16), (18), and (21) into (19) yields the solution of (17) in the strip domain as

$$
\begin{aligned}
g(z)= & \frac{1}{1-\beta} \frac{\left(\mathrm{e}^{\pi z / H}-1\right)^{-1 / 2+\mathrm{i} \varepsilon}\left(\mathrm{e}^{\pi z / H}-\mathrm{e}^{\pi L / H}\right)^{-1 / 2-\mathrm{i} \varepsilon} \mathrm{e}^{\pi z / H}}{2 H \mathrm{i}} \\
& \times \int_{\Gamma} \frac{\sigma_{y y}(t)+\mathrm{i} \sigma_{x y}(t)}{\left(\mathrm{e}^{\pi t / H}-1\right)^{-1 / 2+\mathrm{i} \varepsilon}\left(\mathrm{e}^{\pi t / H}-\mathrm{e}^{\pi L / H}\right)^{-1 / 2-\mathrm{i} \varepsilon}\left(\mathrm{e}^{\pi t / H}-\mathrm{e}^{\pi z / H}\right)} \mathrm{d} t .
\end{aligned}
$$

Consequently, due to $g^{+}(x)=g^{-}(x)=g(x)$ on the bonded portions of the interface and the self-equilibrated traction $\mathbf{T}(x)=p(x)+\mathrm{i} q(x)=-\left(\sigma_{y y}+\mathrm{i} \sigma_{x y}\right)$ acting on the crack surfaces, the SIFs at the left and right crack tips can be evaluated respectively as

$$
\begin{aligned}
\mathbf{K}^{(0,0)}= & \sqrt{2 \pi} \lim _{x \rightarrow 0^{+}} 2 x^{1 / 2-\mathrm{i} \varepsilon}\left(l_{0}\right)^{\mathrm{i} \varepsilon} g(x) \\
= & \sqrt{\frac{2}{H}} \cosh (\pi \varepsilon)\left(\frac{H}{\pi l_{0}}\right)^{-\mathrm{i} \varepsilon}(1-\gamma)^{-1 / 2-\mathrm{i} \varepsilon}\left\{\int_{-\infty}^{0}\left[\frac{1-\gamma \mathrm{e}^{\pi x / H}}{1-\mathrm{e}^{\pi x / H}}\right]^{1 / 2+\mathrm{i} \varepsilon} \mathbf{T}(x) \mathrm{d} x\right. \\
& \left.+\int_{L}^{\infty}\left[\frac{1-\gamma \mathrm{e}^{\pi x / H}}{1-\mathrm{e}^{\pi x / H}}\right]^{1 / 2+\mathrm{i} \varepsilon} \mathbf{T}(x) \mathrm{d} x\right\},
\end{aligned}
$$

and

$$
\begin{aligned}
\mathbf{K}^{(L, 0)}= & \sqrt{2 \pi} \lim _{x \rightarrow L^{-}} 2(x-L)^{1 / 2+\mathrm{i} \varepsilon}\left(l_{0}\right)^{-\mathrm{i} \varepsilon} g(x) \\
= & \sqrt{\frac{2}{H}} \cosh (\pi \varepsilon)\left(\frac{H}{\pi l_{0}}\right)^{\mathrm{i} \varepsilon}\left(\frac{\gamma}{1-\gamma}\right)^{-1 / 2+\mathrm{i} \varepsilon}\left\{\int_{-\infty}^{0}\left[\frac{1-\mathrm{e}^{\pi x / H}}{1-\gamma \mathrm{e}^{\pi x / H}}\right]^{1 / 2-\mathrm{i} \varepsilon} \mathbf{T}(x) \mathrm{d} x\right. \\
& \left.+\int_{L}^{\infty}\left[\frac{\mathrm{e}^{\pi x / H}-1}{\gamma \mathrm{e}^{\pi x / H}-1}\right]^{1 / 2-\mathrm{i} \varepsilon} \mathbf{T}(x) \mathrm{d} x\right\},
\end{aligned}
$$

where

$$
\gamma=\exp (-\pi L / H)
$$

The corresponding ERRs can be obtained by inserting (23) and (24) into (6). 


\section{Examples}

Here we consider two special loading cases with forces only acting at the left crack surfaces: (1) a pair of self-equilibrated forces; (2) self-equilibrated uniform forces. Thus, only the first integral in (23) and (24) contributes the SIF values, and (23) and (24) further reduce to

$$
\mathbf{K}^{(0,0)}=\sqrt{\frac{2}{H}} \cosh (\pi \varepsilon)\left(\frac{H}{\pi l_{0}}\right)^{-\mathrm{i} \varepsilon}(1-\gamma)^{-1 / 2-\mathrm{i} \varepsilon} \int_{-\infty}^{0}\left[\frac{1-\gamma \mathrm{e}^{\pi x / H}}{1-\mathrm{e}^{\pi x / H}}\right]^{1 / 2+\mathrm{i} \varepsilon} \mathbf{T}(x) \mathrm{d} x
$$

and

$$
\mathbf{K}^{(L, 0)}=\sqrt{\frac{2}{H}} \cosh (\pi \varepsilon)\left(\frac{H}{\pi l_{0}}\right)^{\mathrm{i} \varepsilon}\left(\frac{\gamma}{1-\gamma}\right)^{1 / 2-\mathrm{i} \varepsilon} \int_{-\infty}^{0}\left[\frac{1-\mathrm{e}^{\pi x / H}}{1-\gamma \mathrm{e}^{\pi x / H}}\right]^{1 / 2-\mathrm{i} \varepsilon} \mathbf{T}(x) \mathrm{d} x .
$$

\subsection{Isolated forces on surfaces of a semi-infinite crack}

Suppose the left crack is opened by a complex traction $\mathrm{T}=P+\mathrm{i} Q$ at $x=-a$ on each side of the left crack. Its SIFs can be obtained by substituting $\mathbf{T}(x)=(P+\mathrm{i} Q) \delta(x+a)$ into (26) and (27), where $\delta(x)$ is the Dirac's delta function, such that

$$
\mathrm{K}^{(0,0)}=\sqrt{\frac{2}{H}} \cosh (\pi \varepsilon)\left(\frac{H}{\pi l_{0}}\right)^{-\mathrm{i} \varepsilon}[\alpha(1-\gamma)]^{-1 / 2-\mathrm{i} \varepsilon}(P+\mathrm{i} Q)
$$

and

$$
\mathbf{K}^{(L, 0)}=\sqrt{\frac{2}{H}} \cosh (\pi \varepsilon)\left(\frac{H}{\pi l_{0}}\right)^{\mathrm{i} \varepsilon}\left[\frac{\alpha \gamma}{1-\gamma}\right]^{1 / 2-\mathrm{i} \varepsilon}(P+\mathrm{i} Q),
$$

where

$$
\alpha=\left(1-\mathrm{e}^{-\pi a / H}\right) /\left(1-\gamma \mathrm{e}^{-\pi a / H}\right)
$$

As a check, by letting $L \rightarrow \infty, \gamma \rightarrow 0$ and $\alpha \rightarrow 1-\exp (-\pi a / H)$, then (29) tends to zero and (28) covers the SIF of a semi-infinite interfacial crack between two bonded dissimilar elastic strips opened by a pair of self-equilibrated forces $\mathbf{T}(x)=(P+\mathrm{i} Q) \delta(x+a)$ as

$$
\mathbf{K}=\sqrt{\frac{2}{H}} \cosh (\pi \varepsilon)\left(\frac{H}{\pi l_{0}}\right)^{-\mathrm{i} \varepsilon} \frac{P+\mathrm{i} Q}{\left(1-\mathrm{e}^{-\pi a / H}\right)^{1 / 2+\mathrm{i} \varepsilon}},
$$

which agrees with the solution given by $\mathrm{Wu}$ et al. [22].

Furthermore, by letting $H \rightarrow \infty$ and using L'Hopital's rule, results (28) and (29) cover the SIFs for two semi-infinite collinear cracks between two bonded dissimilar half-planes as follows: 


$$
\mathbf{K}^{(0,0)}=\sqrt{\frac{2(a+L)}{\pi a L}} \cosh (\pi \varepsilon)\left[\frac{a L}{(a+L) l_{0}}\right]^{-\mathrm{i} \varepsilon}(P+\mathrm{i} Q)
$$

and

$$
\mathbf{K}^{(L, 0)}=\sqrt{\frac{2 a}{\pi(a+L) L}} \cosh (\pi \varepsilon)\left[\frac{(a+L) L}{a l_{0}}\right]^{\mathrm{i} \varepsilon}(P+\mathrm{i} Q),
$$

which are corresponding to the solutions in the literature [28].

If further letting $L \rightarrow \infty$, solution (33) tends to zero and (32) reduces to

$$
\mathbf{K}=\sqrt{\frac{2}{\pi a}} \cosh (\pi \varepsilon)\left(\frac{a}{l_{0}}\right)^{-\mathrm{i} \varepsilon}(P+\mathrm{i} Q),
$$

which accords to the solution given by Hutchinson et al. [29].

On the other hand, if letting $\varepsilon \rightarrow 0$, results (28) and (29), and (31-34) cover the SIFs for one (two) semi-infinite crack(s) in a homogenous elastic strip or an infinite homogenous elastic plane. The limiting material cases of (32-34) can be found in the SIF handbook by Tada et al. [30].

\subsection{Uniform forces on surfaces of a semi-infinite crack}

Now suppose the left crack is opened by a complex uniform traction $\mathbf{t}=p+\mathrm{i} q$ in the interval $x \in[-a, 0]$ on each side of the crack. In this case, the SIFs can be evaluated by substituting $\mathbf{T}(x)=p+\mathrm{i} q$ into (23) and (24) then integrating with respect to the variable $x$ in the interval $[-a, 0]$ as follows:

$$
\begin{aligned}
\mathbf{K}^{(0,0)} & =\sqrt{\frac{2}{H}} \cosh (\pi \varepsilon)\left(\frac{H}{\pi l_{0}}\right)^{-\mathrm{i} \varepsilon}(1-\gamma)^{-1 / 2-\mathrm{i} \varepsilon}(p+\mathrm{i} q) \int_{0}^{a}\left[\frac{1-\gamma \mathrm{e}^{-\pi x / H}}{1-\mathrm{e}^{-\pi x / H}}\right]^{1 / 2+\mathrm{i} \varepsilon} \mathrm{d} x \\
& =\frac{\sqrt{2 H}}{\pi} \cosh (\pi \varepsilon)\left(\frac{H}{\pi l_{0}}\right)^{-\mathrm{i} \varepsilon}(1-\gamma)^{-1 / 2-\mathrm{i} \varepsilon}(p+\mathrm{i} q)\left[F_{1}\left(\frac{a}{H}, \gamma, \varepsilon\right)+\mathrm{i} F_{2}\left(\frac{a}{H}, \gamma, \varepsilon\right)\right]
\end{aligned}
$$

and

$$
\begin{aligned}
\mathbf{K}^{(L, 0)} & =\sqrt{\frac{2}{H}} \cosh (\pi \varepsilon)\left(\frac{H}{\pi l_{0}}\right)^{\mathrm{i} \varepsilon}\left(\frac{\gamma}{1-\gamma}\right)^{1 / 2-\mathrm{i} \varepsilon}(p+\mathrm{i} q) \int_{0}^{a}\left(\frac{1-\mathrm{e}^{-\pi x / H}}{1-\gamma \mathrm{e}^{-\pi x / H}}\right)^{1 / 2-\mathrm{i} \varepsilon} \mathrm{d} x \\
& =\frac{\sqrt{2 H}}{\pi} \cosh (\pi \varepsilon)\left(\frac{H}{\pi l_{0}}\right)^{\mathrm{i} \varepsilon}\left(\frac{\gamma}{1-\gamma}\right)^{1 / 2-\mathrm{i} \varepsilon}(p+\mathrm{i} q)\left[G_{1}\left(\frac{a}{H}, \gamma, \varepsilon\right)+\mathrm{i} G_{2}\left(\frac{a}{H}, \gamma, \varepsilon\right)\right],
\end{aligned}
$$

where four real coefficients $F_{1}, F_{2}, G_{1}$, and $G_{2}$ are determined by the following integrals as 


$$
\begin{aligned}
& F_{1}\left(\frac{a}{H}, \gamma, \varepsilon\right)=\int_{\mathrm{e}^{-\pi a / H}}^{1} \sqrt{\frac{1-\gamma x}{1-x}} \cos \left[\varepsilon \ln \left(\frac{1-\gamma x}{1-x}\right)\right] \frac{1}{x} \mathrm{~d} x, \\
& F_{2}\left(\frac{a}{H}, \gamma, \varepsilon\right)=\int_{\mathrm{e}^{-\pi a / H}}^{1} \sqrt{\frac{1-\gamma x}{1-x}} \sin \left[\varepsilon \ln \left(\frac{1-\gamma x}{1-x}\right)\right] \frac{1}{x} \mathrm{~d} x, \\
& G_{1}\left(\frac{a}{H}, \gamma, \varepsilon\right)=\int_{\mathrm{e}^{-\pi a / H}}^{1} \sqrt{\frac{1-x}{1-\gamma x}} \cos \left[\varepsilon \ln \left(\frac{1-\gamma x}{1-x}\right)\right] \frac{1}{x} \mathrm{~d} x, \\
& G_{2}\left(\frac{a}{H}, \gamma, \varepsilon\right)=\int_{\mathrm{e}^{-\pi a / H}}^{1} \sqrt{\frac{1-x}{1-\gamma x}} \sin \left[\varepsilon \ln \left(\frac{1-\gamma x}{1-x}\right)\right] \frac{1}{x} \mathrm{~d} x .
\end{aligned}
$$

By letting $L \rightarrow \infty, \gamma \rightarrow 0$, then (36) tends to zero, and (35) reduces to the SIF for a semi-infinite interfacial crack between two bonded dissimilar strips with equal thickness as

$$
\begin{aligned}
\mathbf{K} & =\sqrt{\frac{2}{H}} \cosh (\pi \varepsilon)\left(\frac{H}{\pi l_{0}}\right)^{-\mathrm{i} \varepsilon} \int_{0}^{a} \frac{p+\mathrm{i} q}{\left(1-\mathrm{e}^{-\frac{\pi}{H} x}\right)^{1 / 2+\mathrm{i} \varepsilon}} \mathrm{d} x \\
& =\frac{\sqrt{2 H}}{\pi} \cosh (\pi \varepsilon)\left(\frac{H}{\pi l_{0}}\right)^{-\mathrm{i} \varepsilon}(p+\mathrm{i} q) \sum_{n=0}^{\infty} \frac{\left(1-\mathrm{e}^{-\pi a / H}\right)^{n+1 / 2-\mathrm{i} \varepsilon}}{\left(n+\frac{1}{2}\right)-\mathrm{i} \varepsilon} .
\end{aligned}
$$

If further letting $\varepsilon \rightarrow 0$, result (38) covers the SIF for a semi-infinite crack at the mid-plane of a homogenous elastic strip as

$$
\mathbf{K}=\sqrt{\frac{2}{H}} \int_{0}^{a} \frac{p+\mathrm{i} q}{\left(1-\mathrm{e}^{-\pi x / H}\right)^{1 / 2}} \mathrm{~d} x=\frac{\sqrt{2 H}}{\pi} \ln \left[\frac{1+\sqrt{1-\mathrm{e}^{-\pi a / H}}}{1-\sqrt{1-\mathrm{e}^{-\pi a / H}}}\right](p+\mathrm{i} q),
$$

which agrees with the solution given by Fan [11-13] using Muskhelishvili's potentials [14] combined with conformal mapping technique.

Consequently, if letting $\varepsilon \rightarrow 0$ in (35) and (36), we obtain the SIFs for two semi-infinite cracks at the mid-plane of a homogenous elastic strip as

$$
\begin{aligned}
\mathrm{K}^{(0,0)} & =\sqrt{\frac{2}{H}} \frac{p+\mathrm{i} q}{\sqrt{1-\gamma}} \int_{0}^{a} \sqrt{\frac{1-\gamma \mathrm{e}^{-\pi x / H}}{1-\mathrm{e}^{-\pi x / H}}} \mathrm{~d} x \\
& =\frac{\sqrt{2 H}}{\pi \sqrt{1-\gamma}}\left(\ln \frac{1+\sqrt{\alpha}}{1-\sqrt{\alpha}}-\sqrt{\gamma} \ln \frac{1+\sqrt{\gamma \alpha}}{1-\sqrt{\gamma \alpha}}\right)(p+\mathrm{i} q)
\end{aligned}
$$

and

$$
\begin{aligned}
\mathrm{K}^{(L, 0)} & =\sqrt{\frac{2 \gamma}{H(1-\gamma)}}(p+\mathrm{i} q) \int_{0}^{a} \sqrt{\frac{1-\mathrm{e}^{-\pi x / H}}{1-\gamma \mathrm{e}^{-\pi x / H}}} \mathrm{~d} x \\
& =\frac{\sqrt{2 H}}{\pi \sqrt{1-\gamma}}\left(\sqrt{\gamma} \ln \frac{1+\sqrt{\alpha}}{1-\sqrt{\alpha}}-\ln \frac{1+\sqrt{\gamma \alpha}}{1-\sqrt{\gamma \alpha}}\right)(p+\mathrm{i} q),
\end{aligned}
$$

where $\gamma$ and $\alpha$ are defined in (25) and (30). 
Results (40) and (41) are exactly the solutions obtained recently by Shen and Fan [17]. Discussions of the limiting geometry cases of (40) and (41) can be found in [17].

The ERR of each aforementioned interfacial crack configuration may be easily obtained by inserting (27-29), and (31-39)) into (6).

\section{Conclusion}

In this paper explicit solutions have been obtained for two semi-infinite interfacial cracks between two bonded dissimilar elastic strips depicted in Figure 1. Results (35) and (36) could be used as the surface Green's functions to calculate the SIFs and ERRs of the discussed crack configurations with arbitrary self-equilibrated forces acting on crack surfaces. Combined with conformal mapping technique, the method for Riemann-Hilbert problems has been shown to be a powerful tool in solving some collinear interfacial cracks in strips. The current closed-form solutions can be utilized as a useful theoretical base for the assessment of numerical analysis, especially for estimating the effect of the ratios $a / H, L /$ $H$ and oscillatory index $\varepsilon$ on the SIFs and ERRs in bonded bimaterial structures.

\section{Acknowledgements}

The authors are grateful to the anonymous reviewers from International Journal of Engineering Science for their careful reading and helpful suggestions. Partial support of this work by the U.S. Air Force Office of Scientific Research and the U.S. Army Research Office is gratefully acknowledged. X.-F. Wu would like to express his grateful acknowledgement to the Milton E. Mohr Research Fellowship of the Engineering College at University of Nebraska-Lincoln, 2001, 2002.

\section{References}

1. M. L. Williams, The stress around a fault or crack in dissimilar media. Bull. Seismol. Soc. Am. 49 (1959), pp. 199-204.

2. A. H. England, A crack between dissimilar media. ASME J. Appl. Mech. 32 (1965), pp. 400-402.

3. F. Erdogan, Stress distribution in bonded dissimilar materials with cracks. ASME J. Appl. Mech. 32 (1965), pp. 403-410.

4. J. R. Rice and G. C. Sih, Plane problems of cracks in dissimilar media. ASME J. Appl. Mech. 32 (1965), pp. 418-423.

5. G. P. Cherepanov, Mechanics of Brittle Fracture, McGraw-Hill, New York (1979).

6. J. R. Rice, Elastic fracture mechanics concepts for interfacial cracks. ASME J. Appl. Mech. 55 (1988), pp. 98-103.

7. J. R. Rice, Discussion on the paper: Stress in an infinite strip containing a semi-infinite crack by W. G. Knauss. ASME J. Appl. Mech. 34 (1967), pp. 248-249.

8. H. G. Georgiadis, Complex-variable and integral transform methods for elastodynamic solutions of cracked orthotropic strips. Eng. Fract. Mech. 24 (1986), pp. 727-735.

9. X.-F. Li, Closed-form solution for a mode-III interface crack between two bonded dissimilar elastic layers. Int. J. Fract. 109 (2001), pp. L3-L8. 
10. X.-F. Wu and Y. A. Dzenis, Closed-form solution for a mode-III interfacial edge crack between two bond dissimilar elastic strips. Mech. Res. Commun. 29 (2002), pp. 407-412.

11. T.-Y. Fan, Exact solution of semi-infinite crack in a strip. Chin. Phys. Lett. 7 (1990), pp. 402-405.

12. T.-Y. Fan, Stress intensity factors of mode-I and mode-II for an infinite crack in a strip. Int. J. Fract. 46 (1990), pp. R11-R16.

13. T.-Y. Fan, The Dugdale model for semi-infinite crack in a strip. Eng. Fract. Mech. 37 (1990), pp. 1085-1087.

14. N. I. Muskhelishvili, Some Basic Problems of the Mathematical Theory of Elasticity, P. Noordoff Ltd., Groningen, Holland (1953).

15. T.-Y. Fan, Mathematical modeling on instability of a shear fault. Sci. China (Series E) 26 (1996), pp. 202-209.

16. T. Maruyama and T.-Y. Fan, A breakdown zone model of a fault in a strip. J. Phys. Earth 41 (1993), pp. 21-39.

17. D.-W. Shen and T.-Y. Fan, Exact solutions of two semi-infinite collinear cracks in a strip. Eng. Fract. Mech. 70 (2003), pp. 813-822.

18. Z. Suo and J. W. Hutchinson, Steady-state cracking in brittle substrates beneath adherent films. Int. J. Solids Struct. 25 (1989), pp. 1337-1353.

19. Z. Suo and J. W. Hutchinson, Interface crack between two elastic layers. Int. J. Fract. 43 (1990), pp. 1-18.

20. Z. Suo, Delamination specimens for orthotropic materials. ASME J. Appl. Mech. 57 (1990), pp. 627-635.

21. G. Bao, S. Ho, Z. Suo, and B. Fan, The role of material orthotropy in fracture specimens for composites. Int. J. Solids Struct. 29 (1992), pp. 1106-1116.

22. X.-F. Wu, E. Lilla, and W.-S. Zou, A semi-infinite interfacial crack between two bonded dissimilar elastic strip. Arch. Appl. Mech. 72 (2002), pp. 630-636.

23. J. Dunurs, Elastic interaction of dislocations with inhomogeneities. In: Mathematics Theory of Dislocations, ASME, New York (1968), pp. 70-115.

24. Z. Suo, Singularities interacting with interfaces and cracks. Int. J. Solids Struct. 25 (1989), pp. 1133-1142.

25. M. Comninou, The interface crack. ASME J. Appl. Mech. 44 (1977), pp. 531-636.

26. M. Comninou, The interface crack with friction in the contact zone. ASME J. Appl. Mech. 44 (1977), pp. 780-781.

27. M. Comninou and D. Schmueser, The interface crack in a combined tension-compression and shear field. ASME J. Appl. Mech. 46 (1979), pp. 345-348.

28. G. C. Sih, Handbook of Stress Intensity Factors, Lehigh University, Pennsylvania, Bethlehem (1973).

29. J. W. Hutchinson, M. E. Mear, and J. R. Rice, Crack paralleling an interface between dissimilar materials. ASME J. Appl. Mech. 54 (1987), pp. 828-832.

30. H. Tada, P. C. Paris, and G. R. Irwin, The Stress Analysis of Cracks Handbook, Del Research Corporation, Hellertown, Pennsylvania (1973). 